\title{
Theremin DIY con Arduino UNO
}

\author{
Salvador Moreno ${ }^{1}$, Miguel Damas ${ }^{1}$, Hector Pomares ${ }^{1}$, and Oresti Banos ${ }^{2}$ \\ 1 Departamento de Arquitectura y Tecnología de Computadores \\ Universidad de Granada \\ 2 Telemedicine Group, University of Twente \\ smoreno94@correo.ugr.es, mdamas@ugr.es, hector@ugr.es, \\ o.banoslegran@utwente.nl,
}

Resumen. El objetivo que se persigue con este artículo es mostrar cómo mediante un trabajo de una asignatura propuesto por un alumno (autor principal del artículo) se puede asimilar el concepto de computación física, que es de suma relevancia en los estudios de Grado en Ingeniería Electrónica Industrial de la Universidad de Granada. Concretamente el proyecto implementa en una placa de Arduino UNO un Theremin. Mediante un sensor ultrasonidos HC-SR04 de bajo coste se obtiene la distancia del Theremin a la mano, y a partir de ésta se calcula una nota a emitir dentro de las escalas musicales disponibles en el diseño. La etapa de sonido analógica está conformada por con un amplificador de sonido de baja potencia PAM8403 y un altavoz de $4 \Omega$. Por último, el control del Theremin está facilitado por un LCD de 16x2 caracteres, conectado vía $\mathrm{I} 2 \mathrm{C}, \mathrm{y}$ tres pulsadores.

Palabras clave: Arduino, audio, electrónica, ultrasonidos, Procesadores Integrados

\begin{abstract}
The main objective of this paper is to expose how some valuable concepts related to physical computation, which are very valuable in the Bachellor's Dregree of Industrial Electronic Engineering, can be tackled through a project proposed by a student (the main author of the paper). This work consists of a Theremin implemented in an Arduino UNO board. A low-cost ultrasonic sensor HC-SR04 measures the distance to the controlling hand, calculating then a single note to emit within the available scales in the design. The analogic sound stage is conformed by a low-power sound amplifier PAM8403 and a $4 \Omega$ speaker. Finally, the Theremin's control is provided by a $16 \times 2 \mathrm{LCD}$, connected via I2C, and three buttons.
\end{abstract}

Keywords: Arduino, audio, electronics, ultrasonic, Integrated Processors

\section{Introducción}

Arduino [4] se ha implantado como una de las herramientas más útiles para aquellos que buscan en la electrónica una manera de llevar a cabo sus proyectos 


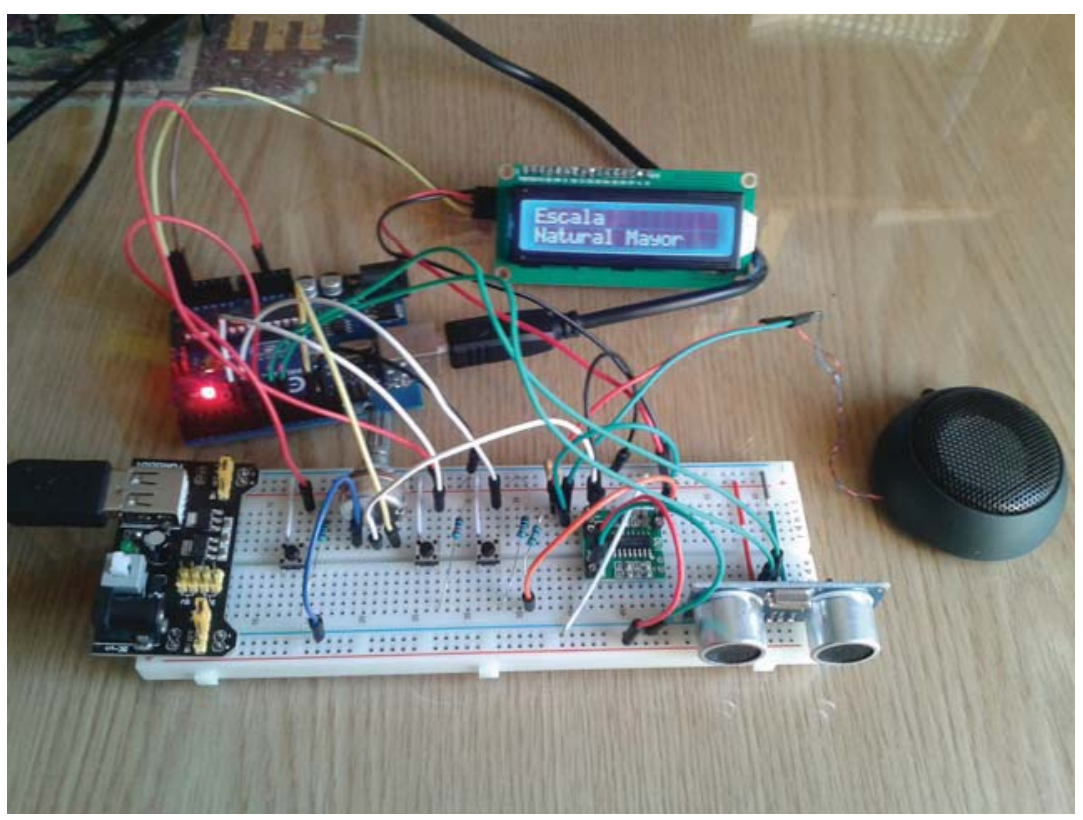

Figura 1. Montaje del dispositivo

y materializar su espíritu creativo. Este proyecto nace como respuesta a una propuesta abierta de trabajo de la titulación de Grado en Ingeniería Electrónica Industrial, concretamente de la asignatura Procesadores Integrados - y de las inquietudes musicales del principal autor del trabajo. Esta asignatura se centra en el estudio de microprocesadores, microcontroladores y procesadores de señales digitales (DSP). Se evalúan las distintas posibilidades que ofrece cada uno de estos componentes, estudiando así diferentes tipos de microcontroladores y aprendiendo a valorar las características necesarias para optimizar nuestros diseños. Además, se analizan los diversos buses y métodos de conexión que se encuentran en la mayoría de los sistemas implantados a día de hoy. Se adquiere así una base con la que el estudiante puede comenzar en relativamente poco tiempo a diseñar sus sistemas, conociendo lo más esencial de cada una de las partes que lo conforman.

El Theremin [1] es uno de los primeros instrumentos musicales electrónicos, desarrollado en 1920. Se compone de dos antenas que, determinando la distancia a la que se encuentra la mano del músico, controlan cada una un oscilador y con él la frecuencia del sonido (que determina la nota musical) y el volumen. Ha sido utilizado por multitud de músicos a lo largo de la historia, de compositores como Percy Graigner o Jean Michael Jarre, llegando incluso a la música popular de manos de grupos como Led Zeppelin o Pink Floyd. La electrónica de este dispositivo es capaz de fascinar a cualquier electrónico, además de lo atractivo 
que se hace como proyecto debido a su índole musical y a que cualquier persona puede interactuar con él.

Se plantea en este trabajo una versión mucho más asequible a nivel de complejidad, así como de presupuesto, como se puede ver en la Figura 1. Buscaremos esa detección de la posición desde la mano hasta nuestro Theremin mediante sensores ultrasonidos de bajo coste como el HC-SR04, adaptando las características del sistema a las limitaciones inherentes al mismo. Se prescinde así de la electrónica compleja de las antenas y osciladores para verse sustituida por un tratamiento de datos y de las señales óptimo, concedidas por la unión del sensor de ultrasonidos con el Arduino UNO. En la bibliografía [2, 3] se pueden encontrar implementaciones más sofisticadas que se siguen sirviendo de Arduino para recoger la señal de los osciladores.

En el marco que proporciona la asignatura de Procesadores Integrados se busca con este proyecto adquirir competencias específicas relacionadas con el uso de microcontroladores (ATMega328P incluido en Arduino UNO), la programación en $\mathrm{C}++$, el procesamiento de señales recogidas con un sensor o transductor (sensor ultrasonidos HC-SR04), la conexión entre elementos del sistema y el mostrar resultados (a través de un LCD de 16x2 conectado por I2C [7]).

\section{Esquema y descripción del Theremin en Arduino}

Entre la variedad disponible de productos Arduino se escoge el UNO ya que buscamos la máxima simplicidad en nuestro Theremín, y que las características del ATMega328P que lo gobierna son óptimas para el control de las señales de ultrasonidos, la emisión de sonido y el funcionamiento del LCD. Las interrupciones del microcontrolador serán las que comanden el sonido y la recepción de ultrasonidos de manera precisa.

\subsection{Medida de distancia con el sensor HC-SR04}

El sensor ultrasonidos HC-SR04 se trata de un medidor de distancia de bajo coste y de funcionalidad limitada. Para su implementación se ha utilizado la librería NewPing [5] (mejorando la oficial Ping), que, sirviéndose del Timer 2 realiza una mejor medida. Además se ha implementado una media móvil de 5 muestras para suavizar la respuesta del dispositivo, mientras se mantiene una sensibilidad adecuada a los cambios de la posición de las manos. Cabe destacar que las capacidades de este sensor están muy restringidas. Aunque aseguran una precisión de hasta $3 \mathrm{~mm}$, las pruebas no otorgan una resolución más allá de $1 \mathrm{~cm}$, en los mejores casos. Estas medidas se realizaron para variaciones de posición relativamente rápidas, siempre utilizando superficies planas para la detección en lugar de la mano. Esto ha impedido implementar un rango de frecuencias de sonido continuo como hace un auténtico Theremin. En su defecto, se ha optado por la discretización de las notas, cada una con su frecuencia asignada, en distintas escalas como solución más plausible, facilitando así la utilización musical del mismo. Se han implementado cinco octavas consecutivas 


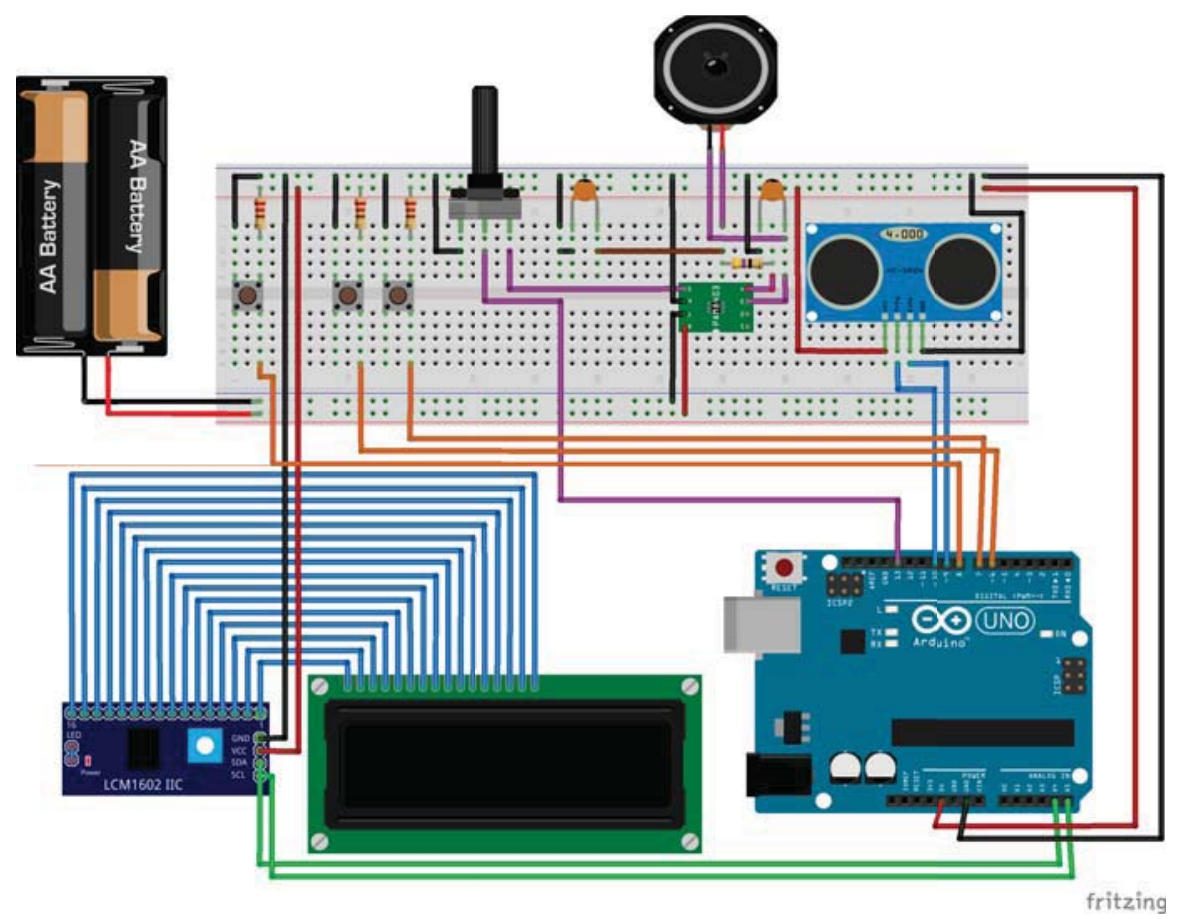

Figura 2. Diagrama de conexión

en el intervalo 3-7 con nota base Do. Las escalas musicales son la cromática, natural mayor y menor, y pentatónicas mayor y menor.

\subsection{Control del Theremín}

Se disponen tres pulsadores en configuración pull-up con resistencias de 10 $k \Omega$ para control del Theremín:

- Intro - ON/OFF

- Más $(+)$

- Menos (-)

Nos servirán para movernos por el menú de selección de escala. Una vez seleccionada, el primer botón (Intro) nos servirá para iniciar el sonido del Theremin. Mientras el dispositivo esté sonando, podremos aumentar o disminuir la octava en las que el programa selecciona las notas con los botones $(+)$ y (-). Pulsando de nuevo el botón ON/OFF paramos la reproducción de sonido.

\subsection{Etapa de audio con amplificador PAM8403}

Para emitir nuestro sonido utilizamos el amplificador de audio PAM8403 y así conectar nuestro altavoz de $4 \Omega$. La conexión de esta etapa viene implementada 


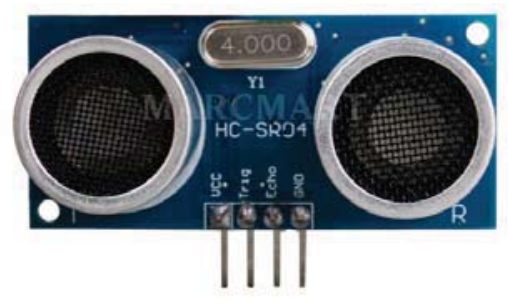

Figura 3. Sensor ultrasonidos HCSR04
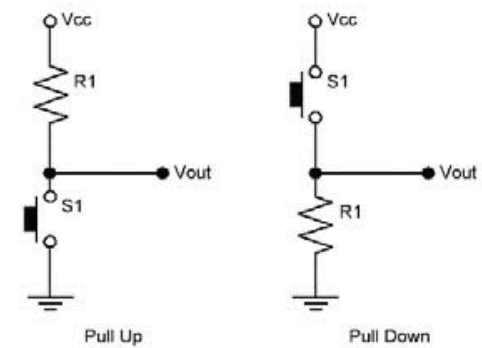

Figura 4. Pulsador en pull-up y en pull-down

para facilitar la adaptación de un altavoz de mayor potencia (hasta $3 \mathrm{~W}$ ) e incluso una posible adición de sonido estéreo. Para este caso hemos utilizado únicamente un canal (mono). Añadimos también 2 condensadores cerámicos 0.1 $\mathrm{uF}$ para filtro de ruido de la etapa por alimentación y una resistencia de $47 \Omega$ en serie con el altavoz para protegerlo, ya que está diseñado para funcionar a una potencia inferior a $3 \mathrm{~W}$.

Un potenciómetro logarítmico de $10 k \Omega$ realiza el control de volumen. Se ha prescindido de la utilización de otro ultrasonidos para el control de volumen por motivos inherentes a la gestión de interrupciones por parte de Arduino UNO, insuficientes para controlar a la vez dos HC-SR04 y la emisión de sonido.

Para la alimentación de esta etapa ha sido necesario un módulo de carga a $5 \mathrm{~V} / 3.3 \mathrm{~V}$. A efectos de test puede utilizarse un altavoz piezoeléctrico en lugar de toda la etapa analógica de amplificación. El sonido se envía a la etapa mediante la NewTone [6](mejorando la librería Tone por defecto) que nos permite seleccionar la frecuencia de exacta del tono que queremos utilizando el Timer 1.

\subsection{Monitorización}

Display. Se utiliza un LCD de 16x2 para visualizar resultados junto con su módulo LCD1602 para controlarlo vía I2C. El control ha sido realizado cómodamente con la librería LiquidCrystal_I2C. La utilización de un módulo I2C está justificada por la gran reducción de número de pines a utilizar. El LCD requiere 6 pines de control más 2 de alimentación, y añadiendo el controlador I2C lo reducimos a 2 pines de control más 2 de alimentación. Ésto es útil sobre todo para una implementación en Arduinos de pequeño tamaño como el UNO.

Watchdog. El watchdog está programado para reiniciar la placa de Arduino en caso de que exista un bloqueo de más de un segundo. El tiempo asignado es alto ya que las pulsaciones vienen acompañadas de delay(100) para evitar rebotes. Luego, puesto que realizamos una media de las últimas 5 muestras del 
ultrasonidos, éstas nos ocupan unos 5x33ms, es decir, 165ms como mínimo de interacción con el HC-SR04. Esto sumado a otros procesos del programa hacen que 500ms o menos no sean suficientes para la programación del watchdog.

\section{Materiales y Presupuesto}

Los componentes necesarios vienen dispuestos en la Tabla 1. El potenciómetro es logarítmico porque el sonido también es percibido de la misma manera (en dB). Las resistencias escogidas son de $1 / 6$ Watt de potencia para asegurar su correcto funcionamiento según la potencia del diseño. El presupuesto total ha sido de $58,35 €$.

\begin{tabular}{|l|l|r|r|r|}
\hline \multicolumn{1}{|c|}{ Componente } & Proveedor & \multicolumn{1}{c|}{ Ud } & Precio/Ud [€/Ud] & Coste [€] \\
\hline Potenciómetro - Rotary Pot 10k Ohm, Log & sparkfun.com & 1 & $2,35 €$ & $2,35 €$ \\
\hline Sensor de distancia ultrasonidos - HC-SR04 & dx.com & 1 & $2,05 €$ & $2,05 €$ \\
\hline Pulsador - Mini Pushbutton Switch & sparkfun.com & 3 & $0,29 €$ & $0,87 €$ \\
\hline Resistor 1k Ohm 1/6 Watt PTH & sparkfun.com & 3 & $0,21 €$ & $0,63 €$ \\
\hline Resistor 47 Ohm 1/6 Watt PTH & sparkfun.com & 1 & $0,21 €$ & $0,21 €$ \\
\hline PAM8403 DC 5V & dx.com & 1 & $1,20 €$ & $1,20 €$ \\
\hline Condensador cerámico 0.1uF & sparkfun.com & 2 & $0,21 €$ & $0,42 €$ \\
\hline Altav0z 4-8 Ohm 0.5W & sparkfun.com & 1 & $1,62 €$ & $1,62 €$ \\
\hline Arduino UNO & sparkfun.com & 1 & $20,79 €$ & $20,79 €$ \\
\hline Módulo LCD I2C - LCD1602 & dx.com & 1 & $1,67 €$ & $1,67 €$ \\
\hline Basic LCD 16x2 & sparkfun.com & 1 & 12,46 & $12,46 €$ \\
\hline Altavoz piezoeléctrico & sparkfun.com & 1 & 1,62 & $1,62 €$ \\
\hline Breadboard Power Supply Stick 5V/3.3V & sparkfun.com & 1 & 12,46 & $12,46 €$ \\
\hline \multicolumn{2}{|c|}{ COSTE TOTAL } & & $\mathbf{5 8 , 3 5}$ \\
\hline
\end{tabular}

Tabla 1. Presupuesto

\section{Conclusiones}

En este trabajo se ha desarrollado un instrumento musical basado en los principios del Theremin e implementado en Arduino UNO con un bajo presupuesto. Se han adquirido y ampliado competencias muy valiosas dentro del campo de la ingeniería electrónica, como sobre tratado y procesamiento de señales de un sensor ultrasonidos HC-SR04, del que se obtiene, a partir de la distancia medida una nota dentro de una multitud de escalas y octavas disponibles, y conocimientos entre partes de un sistema, como la comunicación I2C entre Arduino y el LCD. I2C es además un estándar de comunicación que sigue vigente a día de hoy en multitud de sistemas industriales y de consumo.

Existen multitud de mejoras a implementar, como sustituir el potenciómetro del volumen por otro sensor ultrasonidos (para lo cual haría falta actualizar la versión de Arduino Mega), cambiar el modelo de sensor ultrasonidos por otro 
modelo comercial con una mayor resolución, inserción de un modo metrónomo o su implementación como shield de Arduino UNO.

Se ha realizado así un trabajo completo dentro de las competencias de la asignatura Procesadores Integrados del Grado en Ingeniería Electrónica Industrial, donde además de los conocimientos específicos referentes al desarrollo técnico, se han desarrollado una serie de habilidades generales útiles a la hora de realizar el planteamiento, la planificación y la gestión de un proyecto.

\section{Bibliografía}

1. Theremin World. Accedido 14 de abril de 2016. http://www.thereminworld.com/

2. Liu, Tsung-Ching, Shu-Hui Chang y Che-Yi Hsiao. A modified Quad-Theremin for interactive computer music control. International Conference on Multimedia Technology (ICMT), 6179-82, 2011.

3. Gomes, A., D. Albuquerque, G. Campos, y J. Vieira. TheremUS: The Ultrasonic Theremin, 2:779-85, 2009.

4. Arduino - Home. Accedido 14 de abril de 2016. http://www.arduino.cc/.

5. Arduino Playground - NewPing Library. Accedido 14 de abril de 2016. http://playground.arduino.cc/Code/NewPing.

6. Arduino New Tone. Accedido 14 de abril de 2016. https://bitbucket.org/teckel12/arduino-new-tone/wiki/Home.

7. I2C Bus. Accedido 14 de abril de 2016. http://www.i2c-bus.org/. 